Pierre-Alain Duc, Jonathan Braine and Elias Brinks, eds.

\title{
High Resolution Observations of Molecular Gas in the Outflow of M 82
}

\author{
Fabian Walter \\ NRAO, P.O. Box O, Socorro, NM 87801, USA
}

Axel Weiss

IRAM, Avenida Divina Pastora 7, Granada, Spain

Nick Scoville

Caltech, Pasadena, CA, 91125, USA

\begin{abstract}
We present a high-resolution (3.6", $70 \mathrm{pc}) \mathrm{CO}(1-0)$ mosaic of the molecular gas in M 82 covering an area of $2.5^{\prime} \times 3.5^{\prime}(2.8 \mathrm{kpc} \times 3.9 \mathrm{kpc})$ obtained with the OVRO millimeter interferometer. The observations reveal the presence of huge amounts of molecular gas $(>70 \%$ of the total molecular mass, $\mathrm{M}_{\text {tot }} \approx 1.3 \times 10^{9} \mathrm{M}_{\odot}$ ) outside the central $1 \mathrm{kpc}$ disk. Molecular streamers are detected in and below M 82's disk out to distances from the center of $\sim 1.7 \mathrm{kpc}$. Some of these streamers are well correlated with optical absorption features; they form the basis of some of the prominent tidal HI features around M 82. This provides evidence that the molecular gas within M 82's optical disk is disrupted by the interaction with M 81. Molecular gas is found in M 82's outflow/halo, reaching distances up to $1.2 \mathrm{kpc}$ below the plane; CO line-splitting has been detected for the first time in the outflow. The maximum outflow velocity is $\sim 230 \mathrm{~km} \mathrm{~s}^{-1}$; we derive an opening angle of $\sim 55^{\circ}$ for the molecular outflow cone. The total amount of gas in the outflow is $>3 \times 10^{8} \mathrm{M}_{\odot}$ and its kinetic energy is of order $10^{55} \mathrm{erg}$, about one percent of the estimated total mechanical energy input of M 82's starburst. Our study implies that extreme starburst environments can move significant amounts of molecular gas in to a galaxy's halo (and even to the intergalactic medium).
\end{abstract}

\section{Poster}

Given the limited space, only the key figures are presented here. For more information, color figures and a full list of references the interested reader is referred to Walter, Weiss \& Scoville (2002).

\section{References}

Walter, F., Weiss, A., \& Scoville, N. 2002, ApJ, 580, L21 


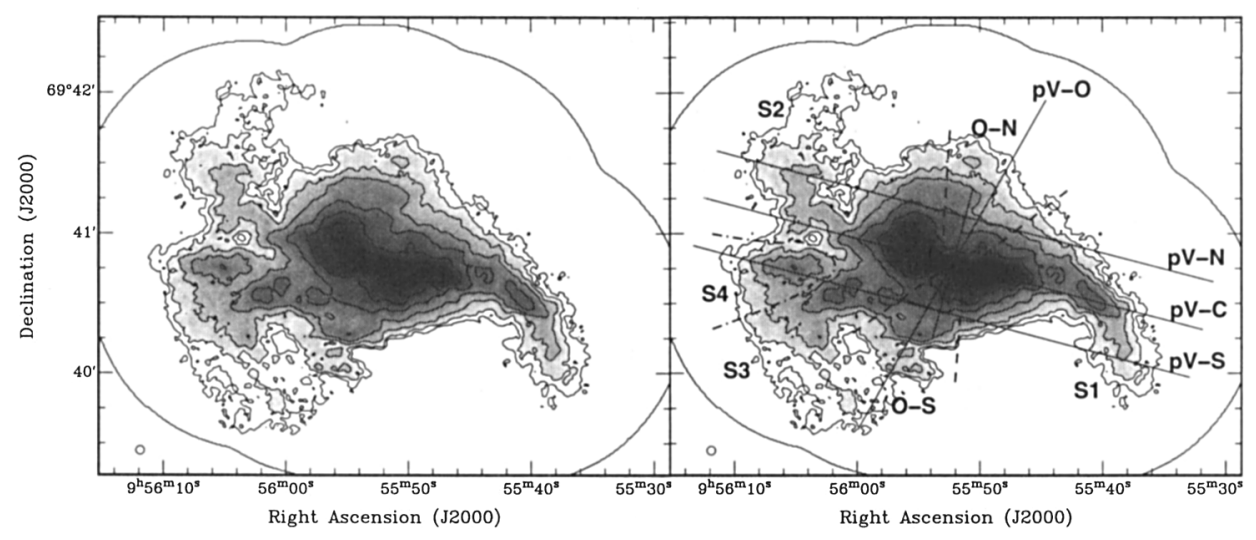

Figure 1. Left: Logarithmic representation of the integrated $\mathrm{CO}(1 \rightarrow 0)$ map of the zero-spacing corrected OVRO mosaic. The OVRO mosaicked field of view is indicated by the outer envelope. Right: Same plot with superimposed labels of the molecular streamers (S1-S4) and the outflow gas (O-N and $\mathrm{O}-\mathrm{S})$. The dashed lines indicate the derived opening angle of the molecular outflow $\left(50^{\circ}\right.$, south; $60^{\circ}$, north, see Walter et al. 2002 for details).
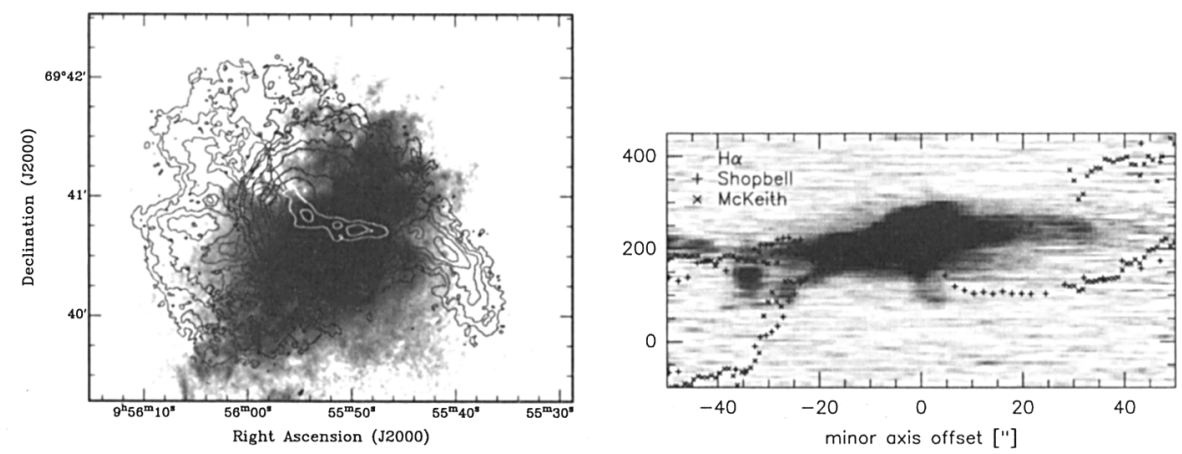

Figure 2. Left: $\mathrm{H} \alpha$ image of M 82 (greyscale). White contours: M 82 disk, Grey contours: M 82 streamer (S1-S4), Black contours: molecular gas associated with the outflow $(\mathrm{O} 1+\mathrm{O} 2)$. Note that molecular gas is clearly associated with M 82's prominent outflow of ionized gas. Right: $\mathrm{CO} \mathrm{pV}$ diagram along the outflow in M $82\left(\mathrm{PA}=150^{\circ}\right.$, see ' $\mathrm{pV}$ $\mathrm{O}^{\prime}$ in Fig. 1 for orientation). The crosses and pluses represent the $\mathrm{H} \alpha$ velocities. Towards the south, CO line splitting is detected. 